\title{
A PARSEVAL-GOLDSTEIN TYPE THEOREM ON THE WIDDER POTENTIAL TRANSFORM AND ITS APPLICATIONS
}

\author{
O. YÜREKLI \\ Department of Mathematics \\ and Computer Science \\ Ithaca College \\ Ithaca, New York 14850 \\ I. SADEK \\ Mathematical Sciences \\ University of North Carolina \\ Wilmington, NC 28403
}

(Received March 19, 1990 and in revised form November 20, 1990)

\begin{abstract}
In this paper a Parseval-Goldstein type theorem involving the Widder potential transform and a Laplace type integral transform is given. The theorem is then shown to yield a relationship between the $\mathcal{K}$-transform and the Laplace type integral transform. The theorem yields some simple algorithms for evaluating infinite integrals. Using the theorem and its results, a number of new infinite integrals of elementary and special functions are presented. Some illustrative examples are also given.
\end{abstract}

KEY WORDS AND PHRASES. The Widder potential transform, the Laplace transform, the $\mathcal{L}_{2}$-transform, the $\mathcal{K}$-transform the modified Bessel function of the third kind or Macdonald's function.

1980 AMS SUBJECT CLASSIFICATION CODES: Primary 4 4A10, 44A15; Secondary S9A40, $44 A 95$.

\section{INTRODUCTION}

Widder $[1,2]$ presented a systematic account of the potential transform

$$
\mathcal{P}[f(x) ; y]=\int_{0}^{\infty} \frac{x f(x)}{x^{2}+y^{2}} d x .
$$

Widder pointed out that the potential transform is related to the Poisson integral representation of a function which is harmonic in a half plane and gave several inversion formulae for the transform and applied his results to harmonic functions. Srivastava and Singh [3] gave the following Parseval-Goldstein type formula:

$$
\int_{0}^{\infty} x \mathcal{P}[f(u) ; x] g(x) d x=\int_{0}^{\infty} x f(x) \mathcal{P}[g(u) ; x] d x
$$

for the Widder potential transform. Srivastava and Yürekli [4] gave the following ParsevalGolstein type relation involving the Laplace transform, the Fourier sine transform and the 
Widder potential transform:

$$
\int_{0}^{\infty} \mathcal{L}[f(u), x] \mathcal{F}_{s}[g(u), x] d x=\int_{0}^{\infty} f(x) \mathcal{P}[g(u), x] d x
$$

where $\mathcal{L}$ is the Laplace transform and $\mathcal{F}_{s}$ is the Fourier sine transform. There are numerous analogous results in the literature on integral transforms. (See, for instance, Goldstein [5], Srivastava [6], Srivastava and Panda [7] and Yürekli [8].)

The objective of this paper is to establish a Parseval-Goldstein type relation between the potential transform and a Laplace type integral transform called $\mathcal{L}_{2}$-transform where the $\mathcal{L}_{2}$-transform is defined as

$$
\mathcal{L}_{2}[f(x) ; y]=\int_{0}^{\infty} x e^{-x^{2} y^{2}} f(x) d x .
$$

If we make a change of variable in the integral on the right-hand side of (1.4), one obtains

$$
\mathcal{L}_{2}[f(x) ; y]=\frac{1}{2} \int_{0}^{\infty} e^{-x y^{2}} f(\sqrt{x}) d x .
$$

Comparing (1.5) with the definition of Laplace transform we obtain the following relationship between the Laplace transform and the $\mathcal{L}_{2}$-transform

$$
\mathcal{L}_{2}[f(x) ; y]=\frac{1}{2} \mathcal{L}\left[f(\sqrt{x}) ; y^{2}\right]
$$

We also obtain identities relating the $\mathcal{K}$-transform

$$
\mathcal{K}_{\nu}[f(x) ; y]=\int_{0}^{\infty} \sqrt{x y} K_{\nu}(x y) f(x) d x
$$

to the $\mathcal{L}_{2}$-transform, where $K_{\nu}(x)$ is the Bessel function of the third kind (it is also known as the Macdonald function), and the Laplace transform to the $\mathcal{L}_{2}$-transform. Using these results we show how one can extend tables of Laplace and Hankel transforms. (See Erdélyi et al. [9, 10], Oberhettinger [11], Oberhettinger and Badii [12].) For definitions of special functions that are used in the paper, the reader is referred to Oldham and Spanier [13], and Erdélyi et al. [14].

We note that if we write $\mathcal{M}[f(x) ; y]=F(y)$ where $\mathcal{M}$ represents any integral transform, we mean the $\mathcal{M}$-transform of $f(x)$ exists and it is $F(y)$.

\section{A PARSEVAL-GOLDSTEIN THEOREM AND ITS COROLLARIES}

LEMMA 2.1. We have

$$
\mathcal{L}_{2}\left[\mathcal{L}_{2}[f(x) ; z] ; y\right]=\frac{1}{2} \mathcal{P}[f(x) ; y],
$$

provided that the integrals involved converge absolutely.

PROOF: Using the definition of the $\mathcal{L}_{2}$-transform we obtain

$$
\mathcal{L}_{2}\left[\mathcal{L}_{2}[f(x) ; z] ; y\right]=\int_{0}^{\infty} z e^{-y^{2} z^{2}}\left(\int_{0}^{\infty} x e^{-x^{2} z^{2}} f(x) d x\right) d z
$$

Changing the order of integration on the right side of (2.2), which is permissible by the absolute convergence of integrals, we have from (2.2) that

$$
\begin{aligned}
\mathcal{L}_{2}\left[\mathcal{L}_{2}[f(x) ; z] ; y\right] & =\int_{0}^{\infty} x f(x)\left(\int_{0}^{\infty} z e^{-\left(x^{2}+y^{2}\right) z^{2}} d z\right) d x \\
& =\frac{1}{2} \int_{0}^{\infty} \frac{x f(x)}{x^{2}+y^{2}} d x
\end{aligned}
$$


Now the result follows from (1.1) and (2.3).

THEOREM 2.1. We have

$$
\int_{0}^{\infty} x \mathcal{L}_{2}[f(y) ; x] \mathcal{L}_{2}[g(z) ; x] d x=\frac{1}{2} \int_{0}^{\infty} y f(y) \mathcal{P}[g(z) ; y] d y
$$

provided that the integrals involved converge absolutely.

PROOF: Using the definition of the $\mathcal{L}_{2}$-transform we obtain

$$
\int_{0}^{\infty} x \mathcal{L}_{2}[f(y) ; x] \mathcal{L}_{2}[g(z) ; x] d x=\int_{0}^{\infty} x \mathcal{L}_{2}[g(z) ; x]\left(\int_{0}^{\infty} y e^{-x^{2} y^{2}} d y\right) d x
$$

Changing the order of integration, which is permissible by the hypothesis, and then using the definition of the $\mathcal{L}_{2}$-transform we find that

$$
\int_{0}^{\infty} x \mathcal{L}_{2}[f(y) ; x] \mathcal{L}_{2}[g(z) ; x] d x=\int_{0}^{\infty} y f(y) \mathcal{L}_{2}\left[\mathcal{L}_{2}[g(z) ; x] ; y\right] d y
$$

Now the assertion follows from Lemma 2.1.

REMARK 2.1. We have

$$
\int_{0}^{\infty} x \mathcal{L}_{2}[g(y) ; x] \mathcal{L}_{2}[f(z) ; x] d x=\frac{1}{2} \int_{0}^{\infty} y g(y) \mathcal{P}[f(z) ; y] d y
$$

since the relation (2.4) is symmetrical with respect to $f$ and $g$. Using the relations (2.4) and (2.7) we obtain the Parseval-Goldstein type formula (1.2). Thus, Theorem 2.1 generalizes relation (1.2).

COROLLARY 2.1. We have

$$
\int_{0}^{\infty} x h(x) \mathcal{L}_{2}[f(z) ; x] d x=\int_{0}^{\infty} y f(y) \mathcal{L}_{2}[h(x) ; y] d y .
$$

provided that the integrals involved converge absolutely.

PROOF: The identity (2.8) follows immediately after letting $h(x)=\mathcal{L}_{2}[g(z) ; x]$ in the relation (2.4).

COROLLARY 2.2. We have

$$
\mathcal{P}\left[\mathcal{L}_{2}[g(u) ; x] ; z\right]=\mathcal{L}_{2}[\mathcal{P}[g(u) ; x] ; z]
$$

provided that the integrals involved converge absolutely.

PROOF: We set $f(y)=e^{-x^{2} y^{2}}$ in Theorem 2.1. Then

$$
\begin{aligned}
\mathcal{L}_{2}[f(y) ; x] & =\int_{0}^{\infty} y e^{-\left(z^{2}+x^{2}\right) y^{2}} d y \\
& =\frac{1}{2\left(z^{2}+x^{2}\right)} .
\end{aligned}
$$

Now the assertion (2.9) follows from (2.4) and (2.10).

THEOREM 2.2. If $\operatorname{Re} \nu \geq-1$

$$
\mathcal{K}_{\nu}\left[y^{\nu+\frac{1}{2}} f(y) ; z\right]=2^{\nu} z^{\nu+\frac{1}{2}} \mathcal{L}_{2}\left[x^{2 \nu-2} \mathcal{L}_{2}\left[f(y) ; \frac{1}{2 x}\right] ; z\right]
$$

provided that the integrals involved are absolutely convergent. 
PROOF: We set $g(u)=u^{\nu} J_{\nu}(z u)$ in Theorem 2.1, where $J_{\nu}$ is the Bessel function of the first kind of order $\nu$. Using relation (1.6) and then making use of the Laplace transform table (see [9, formula (30), p. 185]) we have

$$
\begin{aligned}
\mathcal{L}_{2}\left[u^{\nu} J_{\nu}(z u) ; z\right] & =\frac{1}{2} \mathcal{L}\left[u^{\frac{\nu}{2}} J_{\nu}\left(z u^{\frac{1}{2}}\right) ; x^{2}\right] \\
& =\frac{1}{2}\left(\frac{z}{2}\right)^{\nu} x^{-2 \nu-2} \exp \left(-\frac{z^{2}}{4 x^{2}}\right) .
\end{aligned}
$$

Now in order to evaluate the potential transform of the function $g(u)$ we use Lemma 2.1 and obtain

$$
\mathcal{P}[g(u) ; y]=\left(\frac{z}{2}\right)^{\nu} \mathcal{L}_{2}\left[x^{-2 \nu-2} \exp \left(-\frac{z^{2}}{4 x^{2}}\right) ; y\right] .
$$

The $\mathcal{L}_{2}$-transform on the right-hand side of (2.12) may be evaluated by using the relation (1.6) and then the Laplace transform table (see $[9$, formula (20), p. 146]). Thus

$$
\mathcal{P}[g(u) ; y]=y^{\nu} K_{\nu}(z y) .
$$

Substituting the results (2.12) and (2.14) into (2.4) of Theorem 2.1 gives

$$
\int_{0}^{\infty} y^{\nu+1} K_{\nu}(z y) f(y) d y=\left(\frac{z}{2}\right)^{\nu} \int_{0}^{\infty} x^{-2 \nu-1} \exp \left(-\frac{z^{2}}{4 x^{2}}\right) \mathcal{L}_{2}[f(y) ; x] d x .
$$

Now the assertion follows by making the change of variable $x=t / 2$ and then by using the definitions of the $\mathcal{K}$-transform and the $\mathcal{L}_{2}$-transform.

It is well known that

$$
\mathcal{K}_{\frac{1}{2}}(x)=\mathcal{K}_{-\frac{1}{2}}(x)=\left(\frac{\pi}{2 x}\right)^{\frac{1}{2}} e^{-x},
$$

(see [13, p. 306]). Using (2.11) and (2.16) we obtain the identities in the following corollary: COROLLARY 2.3. We have

$$
\begin{aligned}
& \mathcal{L}[y f(y) ; z]=\frac{2}{\sqrt{\pi}} z \mathcal{L}_{2}\left[\frac{1}{x} \mathcal{L}_{2}\left[f(y) ; \frac{1}{2 x}\right] ; z\right] \\
& \mathcal{L}[y f(y) ; z]=\frac{1}{\sqrt{\pi}} \mathcal{L}_{2}\left[\frac{1}{x^{3}} \mathcal{L}_{2}\left[f(y) ; \frac{1}{2 x}\right] ; z\right] .
\end{aligned}
$$

provided that the integrals involved are absolutely convergent.

\section{EXAMPLES}

We shall illustrate the above results by several examples. In the following example we present a known result (see [10, formula (1), p. 127]) in order to verify relation (2.11).

EXAMPLE 3.1. We show that

$$
\mathcal{K}_{\nu}\left[y^{\rho-1} ; z\right]=2^{\rho-\frac{3}{2}} z^{-\rho} \Gamma\left(\frac{\rho}{2}-\frac{\nu}{2}+\frac{1}{4}\right) \Gamma\left(\frac{\rho}{2}+\frac{\nu}{2}+\frac{1}{4}\right)
$$

provided that $\operatorname{Re} \rho>|\operatorname{Re} \nu|-\frac{1}{2}$.

We set $f(y)=y^{\rho-\nu-\frac{3}{2}}$ in Theorem 3.2. Making use of identity (1.6) we obtain

$$
\begin{aligned}
\mathcal{L}_{2}\left[y^{\rho-\nu-\frac{3}{4}} ; \frac{1}{2 x}\right] & =\frac{1}{2} \mathcal{L}\left[y^{\frac{\rho}{2}-\frac{\nu}{2}-\frac{3}{4}} ; \frac{1}{4 x^{2}}\right] \\
& =2^{\rho-\nu+\frac{1}{2}} \Gamma\left(\frac{\rho}{2}-\frac{\nu}{2}+\frac{1}{4}\right) x^{\rho-\nu-\frac{1}{2}}
\end{aligned}
$$


provided that $\operatorname{Re}(\rho-\nu)>\frac{1}{2}$. Substituting (3.2) into (2.11) we find

$$
\mathcal{K}_{\nu}\left[y^{\rho-1} ; z\right]=2^{\rho}-\frac{1}{2} \Gamma\left(\frac{\rho}{2}-\frac{\nu}{2}+\frac{1}{4}\right) z^{\nu+\frac{1}{2}} \mathcal{L}_{2}\left[x^{\rho+\nu-\frac{3}{2}} ; z\right]
$$

Now formula (3.1) follows after evaluating the $\mathcal{L}_{2}$-transform on the right side of equation (3.3).

Integral transforms evaluated in Examples 3.2, 3.4 and 3.5, and in the appendix, to the best of the author's knowledge, are all new.

EXAMPLE 3.2. We show that

$$
\mathcal{K}_{\nu}\left[y^{\nu+\frac{1}{2}} \sin a y^{2} ; z\right]=a^{\frac{3}{2}}(2 a)^{-\nu-3} \Gamma(\nu+2) z^{\nu+\frac{3}{2}} \mathrm{~S}_{-\nu-\frac{3}{2}, \frac{1}{2}}\left(\frac{z^{2}}{4 a}\right) .
$$

where $\operatorname{Re} \nu>-2$ and $S_{\mu, \nu}$ is the Lommel function.

We set $f(y)=\sin a y^{2}$ in Theorem 3.2. Making use of (1.6) and then tables of Laplace transforms (see [12, formula 7.1, p.54]), we obtain

$$
\begin{aligned}
\mathcal{L}_{2}\left[y^{\nu+\frac{1}{2}} \sin a y^{2} ; \frac{1}{2 x}\right] & =\frac{1}{2} \mathcal{L}\left[\sin a y ; \frac{1}{4 x^{2}}\right] \\
& =\frac{2 \alpha x^{4}}{x^{4}+\alpha^{2}}
\end{aligned}
$$

where $\alpha=1 / 4 a$. Substituting the function $f$ into (2.11), and using (3.5) and (1.6), we find

$$
\mathcal{K}_{\nu}\left[y^{\nu+\frac{1}{2}} \sin a y^{2} ; z\right]=2^{\nu} \alpha z^{\nu+\frac{1}{2}} \mathcal{L}\left[\frac{x^{\nu+1}}{x^{2}+\alpha^{2}} ; z^{2}\right] .
$$

Now (3.4) follows from tables of Laplace transforms (see [12, formula 3.11, p.22]).

Using the technique of Example 3.2, we provide additional results in the appendix.

In the following example we obtain a well known result (Erdélyi $[9$, formula (30) p. 153]) as a special case of Example 3.2.

EXAMPLE 3.3. We show that

$$
\mathcal{L}\left[\sin \left(a y^{2}\right) ; z\right]=\sqrt{\frac{\pi}{2 a}}\left\{\left(\frac{1}{2}-\mathrm{C}(t)\right) \cos t+\left(\frac{1}{2}-\mathrm{S}(t)\right) \sin t\right\},
$$

where $t=z^{2} /(4 a)$, and $\mathrm{C}(t)$ and $\mathrm{S}(\mathrm{t})$ are the Fresnel integrals.

We set $\nu=-1 / 2$ in (3.4). Using (2.16) and the definition of the $\mathcal{K}$-transform we obtain

$$
\mathcal{L}\left[\sin a y^{2} ; z\right]=\frac{z}{4 a} \mathrm{~S}_{-1, \frac{1}{2}}\left(\frac{z^{2}}{4 a}\right) .
$$

It follows from a formula on the Lommel function (see [12, p. 416]) that

$$
\mathrm{S}_{-1, \frac{1}{2}}(t)=\pi\left[\mathrm{J}_{\frac{1}{2}}(t)+\mathrm{J}_{-\frac{1}{2}}(t)-\mathrm{J}_{\frac{1}{2}}(t)-\mathrm{J}_{-\frac{1}{2}}(t)\right],
$$

where $J_{\nu}(t)$ is the Bessel function of order $\nu$ and $J_{\nu}(t)$ is the Anger-Weber function of order $\nu$. However, we have

$$
\mathrm{J}_{\frac{1}{2}}(t)=\sqrt{\frac{2}{\pi t}} \sin t \quad \text { and } \quad \mathrm{J}_{-\frac{1}{2}}(t)=\sqrt{\frac{2}{\pi t}} \cos t
$$


see $[13$, p. 306] and

$$
\begin{aligned}
\mathbf{J}_{\frac{1}{2}}(t) & =\sqrt{\frac{2}{\pi t}}\{[C(t)-\mathrm{S}(t)] \cos t+[\mathrm{C}(t)+\mathrm{S}(t)] \sin t\} \\
\mathbf{J}_{-\frac{1}{2}}(t) & =\sqrt{\frac{2}{\pi t}}\{[\mathrm{C}(t)+\mathrm{S}(t)] \cos t-[\mathrm{C}(t)-\mathrm{S}(t)] \sin t\},
\end{aligned}
$$

see Oberhettinger and Badii [12, p. 415].

Now, substituting (3.10), (3.11) and (3.12) into (3.9) and then using (3.8) we obtain formula (3.7).

EXAMPLE 3.4. We show that

$$
\mathcal{L}\left[\left(x+4 a x^{3}\right)^{\frac{\nu}{2}-\frac{1}{2}} ; z\right]=2^{\nu}(\pi a)^{-\frac{1}{2}} \Gamma\left(\frac{\nu}{2}+\frac{1}{2}\right) z^{12} \exp \left(\frac{z}{8 a}\right) \mathrm{K}_{\frac{\nu}{2}}\left(\frac{z}{8 a}\right),
$$

provided that $-1<\operatorname{Re} \nu<1, \operatorname{Re} a>0$.

We set $f(y)=y^{\nu-1} \exp \left(-a y^{2}\right)$ in Theorem 2.2. Making use of (1.6) and then using tables of Laplace transforms (see [12, formula 5.3, p.37]), we obtain

$$
\begin{aligned}
& \mathcal{L}_{2}\left[y^{-\nu-1} e^{-a y^{2}} ; \frac{1}{2 x}\right]=\frac{1}{2} \mathcal{L}\left[y^{\frac{\nu}{2}-\frac{1}{2}} e^{-a y} ; \frac{1}{4 x^{2}}\right] \\
& =2^{-\nu} \Gamma\left(\frac{1}{2}-\frac{\nu}{2}\right) x^{-\nu}\left(4 a x^{2}+1\right)^{\frac{\nu}{2}-\frac{1}{2}}
\end{aligned}
$$

provided that $\operatorname{Re} \nu<1$. Using tables of Hankel transforms (see [10, formula (24), p. 132]) we obtain

$$
\mathcal{K}_{\nu}\left[y^{-\frac{1}{2}} e^{-a y^{2}} ; z\right]=\frac{1}{4} \sec \left(\frac{\nu \pi}{2}\right)\left(\frac{\pi z}{a}\right)^{\frac{1}{2}} \mathrm{~K}_{\frac{\nu}{2}}\left(\frac{z^{2}}{8 a}\right)
$$

provided that $\operatorname{Re} a>0$ and $-1<\operatorname{Re} \nu<1$. Now formula (3.13) follows from substituting (3.14) and (3.15) into (2.10) and then using (1.6).

EXAMPLE 3.5. We show that

$$
\begin{aligned}
& \mathcal{L}\left[x^{\frac{1}{2}} \operatorname{Erf}\left(a x^{\frac{1}{2}}\right) ; z\right]=\frac{2}{\sqrt{\pi}} z^{-\frac{1}{2}} \arctan \left(a z^{-\frac{1}{2}}\right) \\
& \mathcal{L}\left[x^{\frac{3}{2}} \operatorname{Erf}\left(a x^{\frac{1}{2}}\right) ; z\right]=\frac{4}{\sqrt{\pi}} \arctan \left(a z^{-\frac{1}{2}}\right) .
\end{aligned}
$$

where $\operatorname{Erf}(x)$ is the error function.

We set $f(y)=y^{-2} \sin a y$ in Corollary 2.3. Making use of (1.6) and then tables of integral transforms (see $[12$, formula 7.76, p. 66]) we obtain

$$
\begin{aligned}
\mathcal{L}_{2}\left[\frac{1}{y^{2}} \sin a y ; \frac{1}{2 x}\right] & =\frac{1}{2} \mathcal{L}\left[\frac{1}{y} \sin a y^{\frac{1}{2}} ; \frac{1}{4 x^{2}}\right] \\
& =\frac{\pi}{2} \operatorname{Erf}(a x) .
\end{aligned}
$$

It follows from tables of Laplace transforms (see [12, formula 7.5, p. 54])

$$
\mathcal{L}\left[\frac{1}{y} \sin a y ; z\right]=\arctan \left(\frac{a}{z}\right) .
$$

Now formula (3.16) follows from substituting (3.18) and (3.19) into (2.17). Similarly, formula (3.17) follows from substituting (3.18) and (3.19) into (2.18) of Corollary 2.3. 
ACKNOWLEDGEMENT We would like to thank Thomas K. Boehme for his interest and valuable suggestions. We are also indebted to John Maceli for a careful reading of the preprint. Referee's remarks are also acknowledged.

\section{REFERENCES}

1. D. V. Widder, A transform related to the Poissson integral for a half-plane, Duke Math. J., 33, (1966), 355-362.

2. D. V. Widder, An Introduction to Transform Theory, Academic Press, New York, 1971.

3. H. M. Srivastava and S. P. Singh, A note on the Widder transform related to the Poisson integral for a half-plane, Internat. J. Math. Ed. Sci. Tech., 16, (1985), 675677.

4. H. M. Srivastava and O. Yürekli, A theorem on Widder's potential transform and its applications, J. Math. Anal. and Appl., to appear.

5. S. Goldstein, Operational representations of Whittaker's Confluent Hypergeometric Function and Weber's parabolic function, Proc. London. Math. Soc. (2), 34, (1932), 103-125.

6. H. M. Srivastava, Some theorems on Hardy transform Nederl. Akad. Wetensch. Indag. Math., 30, (1968), 316-320.

7. H. M. Srivastava and R. Panda, Certain multidimensional integral transformations: I and II, Nederl. Akad. Wetensch. Indag. Math., 40, (1978), 118-131 and 132-143.

8. O. Yürekli, A parseval type theorem applied to certain integral transforms, IMA J. Appl. Math., 42, (1989), 241-249.

9. A. Erdélyi et al., Tables of Integral Transforms I, McGraw Hill, New York, 1954.

10. A. Erdélyi et al., Tables of Integral Transforms II, McGraw Hill, New York, 1954.

11. F. Oberhettinger, Tables of Bessel Transforms, Springer-Verlag, New York, 1972.

12. F. Oberhettinger and K. Badii, Tables of Laplace Transforms, Springer-Verlag, New York, (1973).

13. J. Spanier and K. B. Oldham, An Atlas of Functions, Hemisphere Publishing Corporation, New York, 1987.

14. A. Erdélyi et al., Higher Transcendental Functions I, II, III, Springer-Verlag, New York, 1953 and 1954 .

\section{APPENDIX}

\section{A. SOME $\mathcal{K}$-TRANSFORM PAIRS}

The following formulae (A.1) through (A.5) are consequences of Theorem 2.2. The techniques of Example 3.1 and 3.2 are used to obtain these results.

$$
\mathcal{K}_{\nu}\left[y^{\nu+\frac{1}{2}} \sin a y^{2} ; z\right]=2(2 a)^{-\nu+\frac{1}{2}} \Gamma(\nu+2) z^{\nu+\frac{3}{2}} \mathrm{~S}_{-\nu-\frac{3}{2}, \frac{1}{2}}\left(\frac{z^{2}}{4 a}\right)
$$

where $\operatorname{Re} \nu>-2$.

$$
\mathcal{K}_{\nu}\left[y^{\nu+\frac{1}{2}} \cos a y^{2} ; z\right]=2^{-\frac{1}{2}}(2 a)^{-\nu-\frac{1}{2}} \Gamma(\nu+1) z^{\nu+\frac{3}{2}} \mathrm{~S}_{-\nu-\frac{1}{2}, \frac{1}{2}}\left(\frac{z^{2}}{4 a}\right),
$$

where $\operatorname{Re} \nu>-1$.

$$
\mathcal{K}_{1}\left[y^{-\frac{1}{2}} \sin a y^{2} ; z\right]=\frac{1}{2} z^{-\frac{1}{2}}\left[\mathrm{Ci}\left(\frac{z^{2}}{4 a}\right) \sin \left(\frac{z^{2}}{4 a}\right)-\operatorname{si}\left(\frac{z^{2}}{4 a}\right) \cos \left(\frac{z^{2}}{4 a}\right)\right]
$$


where $\mathrm{Ci}(x)$ and $\operatorname{si}(x)$ are cosine and sine integrals, respectively.

$$
\begin{gathered}
\mathcal{K}_{2}\left[y^{-\frac{1}{2}} \sin a y^{2} ; z\right]=z^{-\frac{3}{2}}\left[-\operatorname{Ci}\left(\frac{z^{2}}{4 a}\right) \sin \left(\frac{z^{2}}{4 a}\right)-\operatorname{si}\left(\frac{z^{2}}{4 a}\right) \cos \left(\frac{z^{2}}{4 a}\right)\right]+ \\
\frac{1}{4 a} z^{-\frac{1}{2}}\left[-\operatorname{Ci}\left(\frac{z^{2}}{4 a}\right) \cos \left(\frac{z^{2}}{4 a}\right)-\operatorname{si}\left(\frac{z^{2}}{4 a}\right) \sin \left(\frac{z^{2}}{4 a}\right)\right] . \\
\mathcal{K}_{\nu}\left[y^{\nu+\frac{1}{2}} \sin ^{2} a y^{2} ; z\right]=2^{\nu-4}(2 a)^{-\nu-\frac{3}{2}} \Gamma(\nu+3) z^{\nu+\frac{3}{2}} \mathrm{~S}_{-\nu-\frac{5}{2}, \frac{1}{2}}\left(\frac{z^{2}}{8 a}\right),
\end{gathered}
$$

where $\operatorname{Re} \nu>-3$.

B. SOME LAPLACE TRANSFORM PAIRS

The following formulae (B.1) through (B.5) result from Theorem 2.2 and Corollary 2.3. The techniques of Example 3.4 and 3.5 are used to obtain these results.

$$
\mathcal{L}\left[x^{-2 \mu}\left(4 a x^{2}-x\right)^{\frac{\nu-1}{2}+\mu} ; z\right]=\frac{(4 a)^{\mu}}{z^{\frac{\nu+1}{2}}} \Gamma\left(\frac{\nu+1}{2}-\mu\right) \exp \left(\frac{z}{8 a}\right) \mathrm{W}_{\mu, \frac{\nu}{2}}\left(\frac{z}{4 a}\right)
$$

where $2 \operatorname{Re} \mu<1-|\operatorname{Re} \nu|, \operatorname{Re} a>0$ and $\mathrm{W}_{\mu, \nu}$ is the Whittaker function.

$$
\begin{aligned}
\mathcal{L}\left[x^{\frac{k}{2}+\frac{\mu}{2}-\frac{3}{2}}\right. & \left.\exp \left(\frac{a^{2} x}{2}\right) D_{\nu-\mu-\frac{1}{2}}(a \sqrt{2 x}) ; z\right]= \\
& \frac{\pi^{\frac{1}{2}} 2^{\frac{\mu-\nu}{2}}-\frac{s}{4} \Gamma\left(\mu+\nu+\frac{1}{2}\right)}{\Gamma(\mu+1)\left(a+z^{\frac{1}{2}}\right)^{\mu+\nu+\frac{1}{2}}}{ }_{2} \mathrm{~F}_{1}\left(\mu+\nu+\frac{1}{2}:, \nu+\frac{1}{2}, \mu+1, \frac{a-z^{\frac{1}{2}}}{a+z^{\frac{1}{2}}}\right)
\end{aligned}
$$

where $\operatorname{Re} \mu>|\operatorname{Re} \nu|-\frac{1}{2}, D_{\nu}$ is the parabolic cylinder function and ${ }_{m} F_{n}$ is the hypergeometric function.

$$
\begin{aligned}
\mathcal{L}\left[\frac{\sin \left(\mu \arctan \left(\frac{x}{\alpha}\right)\right)}{x^{\frac{1}{2}}\left(x^{2}+\alpha^{2} x\right)^{\frac{4}{2}}} ; z\right]=\frac{\pi^{\frac{1}{2}} \Gamma(1-\mu)}{4 z^{\frac{\mu}{2}+\frac{1}{2}}} \times \\
\\
\quad\left\{\mathrm{J}_{\frac{1}{2}-\frac{\mu}{2}}\left(\frac{\alpha z}{2}\right) \sin \left(\frac{\alpha z}{2}-\frac{\pi}{2}+\frac{\pi}{4} \mu\right)-Y_{\frac{1}{2}-\frac{\mu}{2}}\left(\frac{\alpha z}{2}\right) \cos \left(\frac{\alpha z}{2}-\frac{\pi}{2}+\frac{\pi}{4} \mu\right)\right\},
\end{aligned}
$$

where $-1<\operatorname{Re} \mu<2$ and $Y_{\nu}$ is the Bessel function of the second kind of order $\nu$.

$$
\mathcal{L}\left[x^{-\frac{1}{2}} \exp \left(\frac{a^{2}}{4 x}\right) \Gamma\left(-\nu, \frac{a^{2}}{4 x}\right) ; z\right]=\pi 2^{2 \nu+2} a^{\frac{1}{2}} z^{-\frac{1}{4}} \Gamma\left(\nu+\frac{3}{2}\right) \mathrm{S}_{-2 \nu-\frac{3}{2}, \frac{1}{2}}\left(a z^{\frac{1}{2}}\right),
$$

where $\operatorname{Re} \nu>-1$.

$$
\mathcal{L}\left[x^{-\frac{3}{2}} \exp \left(\frac{a^{2}}{4 x}\right) \Gamma\left(-\nu, \frac{a^{2}}{4 x}\right) ; z\right]=\pi^{\frac{3}{2}} 2^{2 \nu+3} a^{\frac{1}{2}} \Gamma\left(\nu+\frac{3}{2}\right) \mathrm{S}_{-2 \nu-\frac{3}{2}, \frac{1}{2}}\left(a z^{\frac{1}{2}}\right),
$$

where $\operatorname{Re} \nu>-1$. 


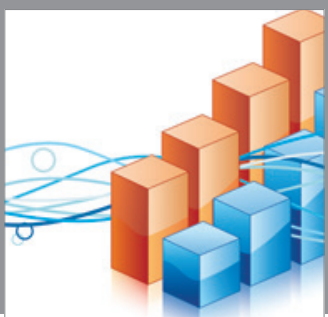

Advances in

Operations Research

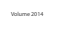

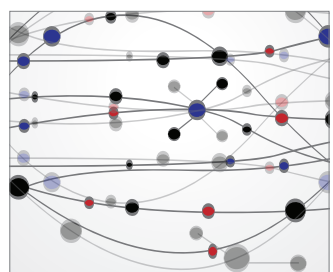

\section{The Scientific} World Journal
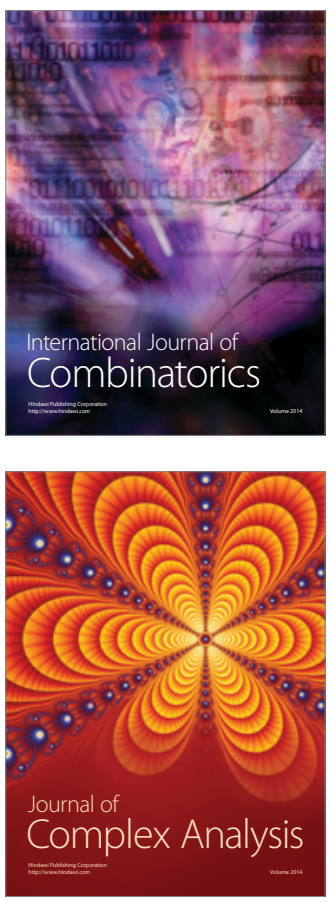

International Journal of

Mathematics and

Mathematical

Sciences
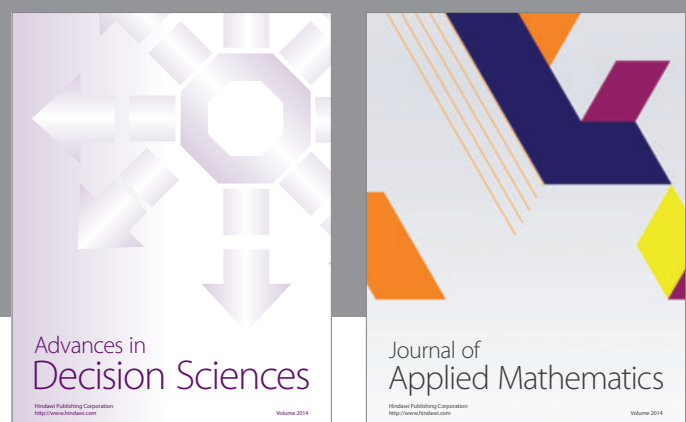

Journal of

Applied Mathematics
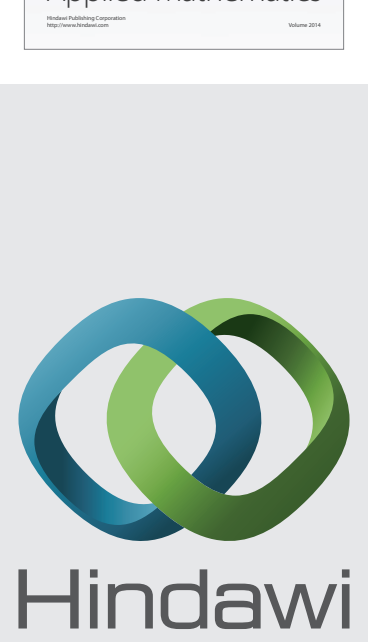

Submit your manuscripts at http://www.hindawi.com
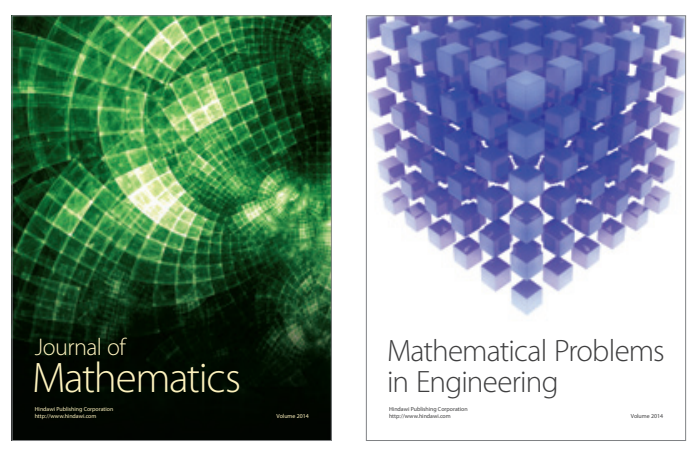

Mathematical Problems in Engineering
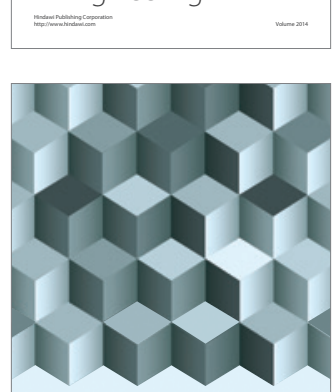

Journal of

Function Spaces
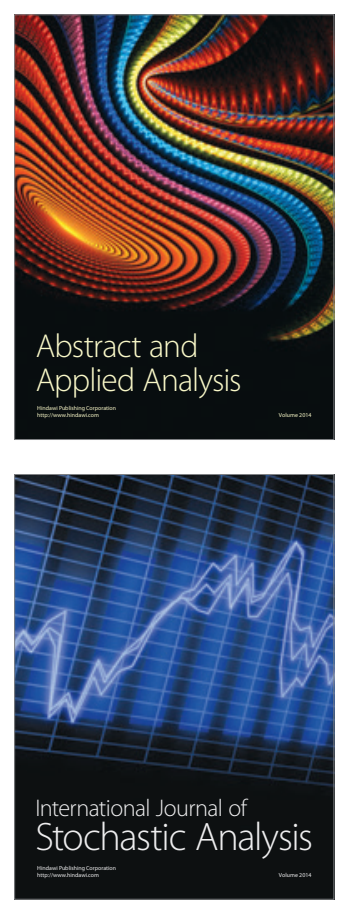

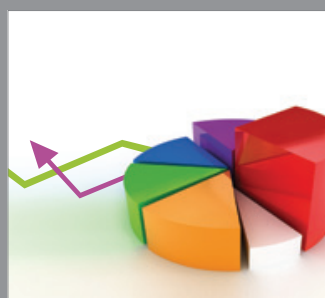

ournal of

Probability and Statistics

Promensencen
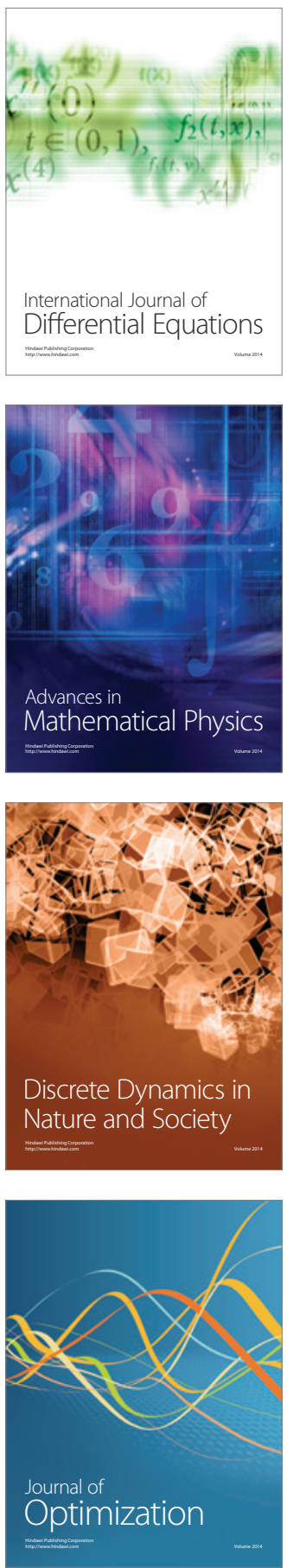